\title{
Một phản ánh giá trị lâu dài của nghiên cứu chính trị-xã hội
}

\author{
Hồ Mạnh Toàn \\ Trung tâm ISR; Trường Đại học Phenikaa \\ Yên Nghĩa, Hà Đông, Hà Nội, Việt Nam \\ Ngày 25 tháng 8 năm 2020
}

ISR | Phenikaa Uni (25/8/2020; https://isr.phenikaa-uni.edu.vn/chitiet/sukien 2019222759 334/mot-phan-anh-gia-tri-lau-dai-cua-nghien-cuu-chinh-tri-xahoi) - Trong hoạt động nghiên cứu khoa học xã hội và chính trị, về cơ bản ảnh hưởng của tác phẩm cũng được đánh giá tương tự như với các sản phẩm nghiên cứu của khoa học tự nhiên. Cụ thể là việc trích dẫn trong các tác phẩm của đồng nghiệp quốc tế, và gần đây, trích dẫn ấy còn được đánh giá sâu hơn qua dữ liệu các hệ thống lưu trữ thư viện học thuật như Scopus và ISI Web of Science.

Bên cạnh đó, nghiên cứu khoa học xã hội, kinh tế và chính trị còn có một góc đánh giá rất quan trọng khác nữa đối với mức độ ảnh hưởng của tác phẩm. Đó chính là các phân tích và quyết định chính sách. Các phân tích và chính sách càng ở tầm mức quốc tế, với các sự kiện có ảnh hưởng rộng lớn, thì việc sử dụng tư liệu học thuật thường sẽ càng "khắt khe" hơn.

Thông thường, hay có quan niệm rằng các nghiên cứu học thuật mới mẻ, thời đại và phản ánh thời sự tức thời sẽ hấp dẫn các phân tích chính sách đương đại. Tuy nhiên, không phải lúc nào quan niệm này cũng chính xác.

Tháng 8-2020, cơ quan nghiên cứu chiến lược quốc tế hàng đầu thế giới của Mỹ-The RAND Corporation-vừa xuất bản cuốn sách có tựa đề North Korean Decisionmaking: Economic Opening, Conventional Deterrence Breakdown, and Nuclear Use.[1] Đối với chính trị quốc tế, và đặc biệt là chính trị nội bộ Mỹ, quan hệ ngoại giao-chính trị-quân sự với Bắc Triều Tiên là một vấn đề gai góc, dai dẳng và khó giải quyết. Mỗi thời kỳ tranh cử, vấn đề này lại nổi lên, và nay khi cuộc bầu cử tổng thống Mỹ sắp bắt đầu, phân tích chính trị này ra đời. Nó chắc chắn sẽ thu hút các nhóm cố vấn chính trị của các ứng cử viên chính trường Mỹ. 


\section{North Korean Decisionmaking}

Economic Opening, Conventional Deterrence Breakdown, and Nuclear Use

by John V. Parachini, Scott W. Harold, Gian Gentile, Derek Grossman, Leah Heejin Kim, Logan Ma, Michael J. Mazarr, Linda Robinson

Related Topics: China, Communism, Cuba, Economic Policy, International Trade

Major Combat Operations, North Korea, Nuclear Deterrence, South Korea, United States, Vietnam

目 Citation 〈 Embed

() $y$ in

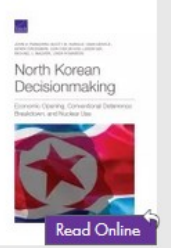

DOWNLOAD EBOOK FOR FREE

Format File Size Notes

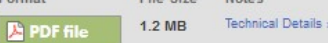

This report is a compilation of three papers designed to stimulate discussion among those who are focused on North Korean decisionmaking. The first paper describes the experiences of North Korea and three similar authoritarian regimes - China, Vietnam, and Cuba - and provides a forecast of why and how North Korea might adopt a new economic model. The second paper describes decisions that the North Korean leadership might face in two scenarios in which conventional deterrence on the Korean Peninsula breaks down. The final paper provides an assessment of North Korean leadership decisionmaking about nuclear weapons doctrine. Despite the many unknowns surrounding the North Korean leadership decisionmaking process,

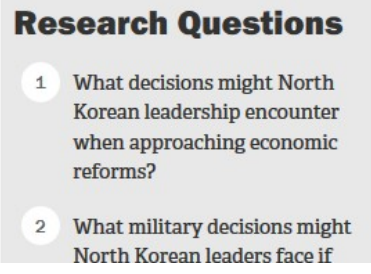

Document Details Copyright: RAND Corporation Avallabillty: Available Print Format: Paperback Paperback Pages: 124 List Price: $\$ 24.00$ Paperback Price: $\$ 19.20$ Paperback ISBN/EAN: 978197740553 DOI: https//doiog/

North Korean Decisionmaking; ISBN: 9781977405531 [1]

Tuy nhiên, điều đáng chú ý là hai trong số các tư liệu học thuật mà cuốn sách chính trịquân sự-ngoại giao mới xuất bản trên sử dụng là hai nghiên cứu đã xuất bản khá lâu của TS. Vương Quân Hoàng, Trung tâm ISR, cùng cộng sự từ cách đây nhiều năm.[2,3]

Thực tế này cho thấy một phản ánh giá trị lâu dài của nghiên cứu chính trị-xã hội, cho dù thời gian đã trôi qua, và thông tin tưởng chừng như đã cũ.

Đồng thời, những người nghiên cứu KHXH cũng có thêm một góc nhìn chân thực về cách đánh giá ảnh hưởng của thông tin học thuật, không chỉ giới hạn trong phạm vi các CSDL học thuật quen dùng ngày nay như Scopus của Elsevier hay ISI Web of Science của Clarivate Analytics.

Bản thân một trong hai tài liệu được trích dẫn cũng là một bài nghiên cứu rút gọn có hình thức như một báo cáo phân tích phổ thông, được xuất bản trên hệ thống Stratfor chuyên phân tích thông tin địa chính toàn cầu, https://worldview.stratfor.com.

Trong hình dưới đây là phần trích dẫn hai tài liệu cũ như vừa nói ở trên trong cuốn North Korean Decisionmaking của The RAND Corp. 
'North Korea's Central Broadcaster: 'Kim Jong-Un Inspects Sinuiju and Maps Construction Plans' (눅한 중앙방송 “김정은, 신의주 시찰하고 건설계흭 지도)," Mackyung Economics, November 16, 2018. As of June 15, 2020: https://www.mk.co.kr/news/politics/view/2018/11/718593/

"North Korean Defector Says Even a Limited Attack by U.S. Would Trigger AllOut War," PBS NewsHour, November 3, 2017. As of June 15, 2020:

https://www.pbs.org/newshour/show/ north-korean-defector-says-even-a-limited-attack-by-u-s-would-trigger-all-out-war

O'Neil, Andrew, "North Korea's Changing Nuclear Posture," The Strategist, April 17, 2013.

Pearson, James, and Ju-min Park, "The War That Never Ends Between the

Koreas," Reuters, June 16, 2014. As of March 16, 2020:

https://www.reuters.com/article/us-northkorea-islands-insight/ the-war-that-never-ends-between-the-koreas-idUSKBN0ES00720140617

Pollack, Jonathan D., The Sino-Soviet Rivalry and Chinese Security Debate, Santa Monica, Calif: RAND Corporation, R-2907-AF, 1982. As of June 8, 2020: https://www.rand.org/pubs/reports/R2907.html

Public Law 106-387, Agriculture, Rural Development, Food and Drug Administration Appropriations Act, October 28, 2000.

Public Law 111-8, Omnibus Appropriations Act, March 11, 2009.

Quan Hoang Vuong, "Vietnam's Political Economy in Transition (1986-2016)," Stratfor, May 27, 2014. As of March 28, 2019:

https://worldview.stratfor.com/article/

vietnams-political-economy-transition-1986-2016

Quan Hoang Vuong, Dam Van Nhue, Daniel van Houtte, and Tri Dung Tran, “The Entrepreneurial Facets as Precursor to Vietnam's Economic Renovation in 1986," IUP Journal of Entrepreneurship Development, Vol. VIII, No. 4, December 2011, pp. 6-47. As of March 26, 2019:

https://papers.ssrn.com/sol3/papers.cfm?abstract_id=2141196

"Report of the Fifth Plenary Meeting of the 7th Central Committee of the WPK

Hai tài liệu cũ được sử dụng [2,3]

Một mặt, các nhà nghiên cứu thường cảm thấy hạnh phúc khi ấn phẩm của mình có tác động tới suy nghĩ và tác phẩm tương lai của một số hay nhiều đồng nghiệp, thế hệ kế tiếp. Mặt khác, trong lĩnh vực chính trị xã hội, chúng ta không xa lạ với những tư tưởng và tác phẩm có ảnh hưởng tới việc hình thành các chính sách, và tác động của chúng đối với nhiều con người, xã hội còn sâu rộng và vượt trội so với những tác động và ảnh hưởng học thuật đơn thuần.

Do đó, qua một việc cụ thể này, các nghiên cứu viên Trung tâm ISR có thêm thông tin khách quan về cách thức sử dụng thông tin học thuật, và có quan niệm cởi mở hơn so với việc thuần túy giới hạn trong cách đo đếm bài báo và trích dẫn của những hệ thống 
tiêu chuẩn như Scopus hay Web of Science. Điều này sẽ dẫn tới những quyết định điều chỉnh về tính đa dạng của sản phẩm, cũng như hệ thống xuất bản, và thể loại thông tin nghiên cứu trong tương lai.

\section{Tài liệu tham khảo:}

[1] John V. Parachini, Scott W. Harold, Gian Gentile, Derek Grossman, Leah Heejin Kim, Logan Ma, Michael J. Mazarr, and Linda Robinson. (2020). North Korean Decisionmaking: Economic Opening, Conventional Deterrence Breakdown, and Nuclear Use. Santa Monica, CA: The RAND Corp. ISBN: 978-1-9774-0553-1.

[2] Quan Hoang Vuong. (2014). Vietnam's Political Economy in Transition (1986-2016). Stratfor, May 27, 2014. URL: https://worldview.stratfor.com/article/vietnamspolitical-economy-transition-1986-2016.

[3] Quan Hoang Vuong, Dam Van Nhue, Daniel van Houtte, and Tri Dung Tran. (2011). The Entrepreneurial Facets as Precursor to Vietnam's Economic Renovation in 1986. The IUP Journal of Entrepreneurship Development, Vol. VIII, No. 4, pp. 6-47. URL: https://papers.ssrn.com/sol3/papers.cfm?abstract id=2141196. 